\title{
Impact des Décharges Sauvages des Déchets Solides sur les Sols à Cotonou
}

\author{
Chérifatou Iyabo Biaou, \\ Laboratoire Pierre PAGNEY "Climat, Eau, \\ Ecosystèmes et Développement" Abomey-Calavi, Bénin \\ Sidonie Clarisse Hedible, \\ Département de Socio-Anthropologie, Faculté des Sciences Humaines et \\ Sociales, Université d'Abomey-Calavi, Bénin \\ Rodrigue Codjo Landeou, \\ Centre de Recherche Entomologique de Cotonou, Bénin \\ Michel Boko, \\ Laboratoire Pierre PAGNEY "Climat, Eau, \\ Ecosystèmes et Développement" Abomey-Calavi, Bénin
}

Doi:10.19044/esj.2019.v15n30p94 URL:http://dx.doi.org/10.19044/esj.2019.v15n30p94

\section{Résumé}

La mauvaise gestion des déchets solides ménagers demeure un problème environnemental majeur pour la ville de Cotonou. De mauvaises pratiques, notamment la mise en décharge sauvage, sont très courantes. L'objectif de cette recherche est de mettre en évidence l'impact qu'ont les décharges sauvages des déchets solides sur les sols à Cotonou. Pour y parvenir, une évaluation des sols de trois décharges non contrôlées a été effectuée par dosage d'éléments traces métalliques (cadmium, cuivre et plomb) dans 8 échantillons composites de sol prélevés dans les horizons de $10 \mathrm{~cm}, 40 \mathrm{~cm}$ et $1 \mathrm{~m}$. L'analyse des échantillons a révélé que les sols sont contaminés par le cadmium $(1,040-6,520 \mathrm{mg} / \mathrm{kg})$, le cuivre $(402,390-904 \mathrm{mg} / \mathrm{kg})$, le plomb $(20-1774,50 \mathrm{mg} / \mathrm{kg}$ ). Les paramètres chimiques déterminés (matière organique et $\mathrm{pH}$ ) varient entre $7,30 \%$ et $71,70 \%$ pour la matière organique, 6,600 et 6,810 pour le $\mathrm{pH}$. La caractérisation des déchets solides ménagers a été également effectuée afin de connaître la composition des déchets. Les résultats obtenus montrent que les déchets sont riches en déchets putrescibles $(31,6 \%)$, éléments fins $(33,8 \%)$. Les déchets plastiques $(7 \%)$ y sont également importants. On y trouve aussi des déchets ménagers spéciaux (piles, accumulateurs de téléphones portables, médicaments, tubes et ampoules fluorescentes, chargeurs de portable, jouets électriques, vernis à ongles, produits d'hygiène, $1,2 \%$ ). 
Mots clés : Cotonou, Décharges non contrôlées, Déchets solides ménagers, Métaux lourds, Sol

\title{
Impact of the Uncotrolled Landfilling of Solid Waste on Soils in Cotonou city
}

\author{
Chérifatou Iyabo Biaou,
}

Laboratoire Pierre PAGNEY "Climat, Eau, Ecosystèmes et Développement" Abomey-Calavi, Bénin

Sidonie Clarisse Hedible,

Département de Socio-Anthropologie, Faculté des Sciences Humaines et

Sociales, Université d'Abomey-Calavi, Bénin

\section{Rodrigue Codjo Landeou,}

Centre de Recherche Entomologique de Cotonou, Bénin

Michel Boko,

Laboratoire Pierre PAGNEY "Climat, Eau,

Ecosystèmes et Développement" Abomey-Calavi, Bénin

\begin{abstract}
The poor management of solid household waste remains a major environmental problem for the city of Cotonou. Bad practices, including landfilling wild are very common. The aim of this research is to highlight the impact of the uncontrolled landfilling of solid waste on soils in Cotonou city. To achieve this objective, a soil assessment of three landfill sites was carried out by assaying metallic trace elements (cadmium, copper and lead) in 8 composite soil samples collected from $10 \mathrm{~cm}, 40 \mathrm{~cm}$ and $1 \mathrm{~m}$ horizons. Analysis of the samples revealed that the soils are contaminated with cadmium $1.040-6.520 \mathrm{mg} / \mathrm{kg})$, copper $(402.390-904 \mathrm{mg} / \mathrm{kg})$, lead $(20-1774.50$ $\mathrm{mg} / \mathrm{kg}$ ). The chemical parameters determined (organic matter and $\mathrm{pH}$ ) vary between $7.30 \%$ and $71.70 \%$ for organic matter, 6.600 and 6.810 for $\mathrm{pH}$. The characterization of household solid waste was also carried out to determine the composition of the waste. The results obtained show that the waste is rich in putrescible elements (31.6\%) and fine elements (33.8\%). Plastic waste (7\%) is also important. There is also special household waste (batteries, mobile phone batteries, medicines, fluorescent tubes and light bulbs, mobile phone chargers, electric toys, nail polish, hygiene products, $1.2 \%$ ).
\end{abstract}


Keywords : Cotonou city,.Household solid waste, Landfill sites, Metal trace Elements, Soil

\section{Introduction}

La quantité de déchets ménagers a connu au cours des dernières décennies un accroissement en raison de la croissance démographique accélérée. Ce phénomène est plus critique dans les pays en développement qui n'ont pas toujours les moyens nécessaires pour gérer convenablement les déchets (Thonart, Diabate, Hiligsmann \& Lardinois, 2005). C'est le cas du Bénin où toutes les villes sont confrontées à des difficultés de gestion des déchets (Babio, Baloubi \& Houssou, 2016). La ville de Cotonou, capitale économique du Bénin, où s'est déroulée cette recherche, affiche des difficultés à gérer ses déchets solides ménagers. En effet, la population produit en moyenne de 718 tonnes de déchets solides ménagers par jour (Mairie Cotonou, 2008). Cette quantité de déchets solides ménagers produits est mal gérée et compromet la qualité de vie. Une simple observation de la ville témoigne de l'ampleur de ce problème : dépôts anarchiques d'ordures dans les bas-fonds, les rues, les canalisations d'eau, sur les berges des eaux de surfaces, les terrains vides non clôturés La mise en décharge sauvage des déchets est donc une pratique très courante et elle peut être dommageable pour l'environnement et pour la population. Au-delà des nuisances générées (odeurs, fumées, plastiques volants), les décharges sauvages peuvent être sources de pollution des sols et des eaux de la nappe phréatique (Nhari, Sbaa, Vasel, Fekhaoui \& El Morhit, 2014 ; Thonart et al., 2005). En fait ces déchets riches en matière organique contiennent des éléments indésirables tels que les déchets dangereux et les déchets d'équipements électriques et électroniques qui peuvent contenir des éléments traces métalliques (Gbèdo, 2010). Ces éléments traces métalliques ou métaux lourds peuvent s'incorporer dans la chaîne alimentaire ou migrer vers les eaux souterraines (Greffard et al. ; Legret et al ; Mazlani et al. cités par Matech et al., 2014). Parmi ces métaux, certains [Manganèse $(\mathrm{Mn})$, le zinc $(\mathrm{Zn})$, le bore $(\mathrm{Br}$.) et le cuivre $(\mathrm{Cu})$ ] constituent des oligo-éléments bénéfiques aux êtres vivants. Mais ils deviennent toxiques à des concentrations très élevées. D'autres par contre [Cadmium $(\mathrm{Cd})$, le Mercure $(\mathrm{Hg})$ et le Plomb $(\mathrm{Pb})]$ sont toxiques à faibles doses pour de nombreuses espèces animales et végétales, aquatiques et terrestres ((TremelSchaub \& Feix ; Walker et al., cités par Matech et al., 2014). L'objectif de cette recherche est de mettre en évidence les impacts de ces décharges sur les sols à Cotonou. Pour ce faire, une analyse des sols de trois décharges sauvage a été réalisée. La méthodologie suivie, les résultats obtenus et la discussion sont présentés ci-dessous. 


\section{Méthodologie}

\section{Cadre géographique du milieu de recherche}

Situées au Nord de la commune de Cotonou, les trois décharges sauvages étudiées se trouvent à proximité des eaux de surface et font l'objet d'une utilisation actuelle (figure 1).

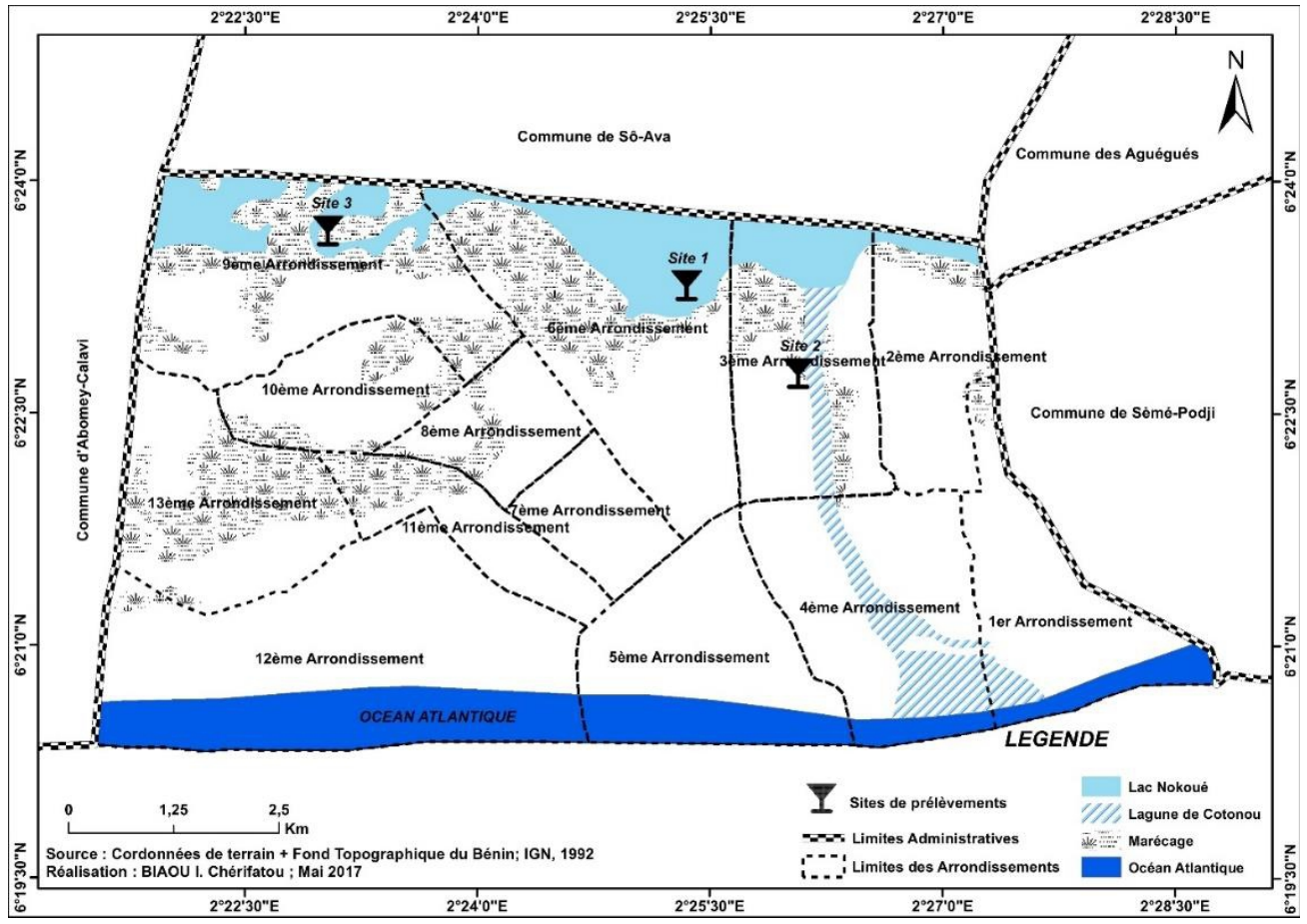

Figure 2 : Situation des sites de prélèvement

La première décharge sélectionnée se trouve dans le $6^{\mathrm{e}}$ (sixième) arrondissement au Nord de la commune de Cotonou. Elle est située à $04^{\circ} 36^{\prime}$ $129^{\prime \prime}$ Nord et $07^{\circ} 06^{\prime} 212^{\prime \prime}$ Est au bord du lac Nokoué et s'étend sur une superficie d'environ $1200 \mathrm{~m}^{2}$.

La deuxième décharge est située dans le $3^{\mathrm{e}}$ (troisième) arrondissement au Nord à $04^{\circ} 37^{\prime} 452^{\prime}$ ' Nord et $07^{\circ} 05^{\prime} 168^{\prime \prime}$ 'Est à proximité du chenal de Cotonou, et s'étend sur une superficie d'environ $800 \mathrm{~m}^{2}$.

La troisième décharge se trouve sur la berge du lac Nokoué à $04^{\circ} 31^{\prime} 863^{\prime}$ ', Nord et $07^{\circ} 06^{\prime} 865^{\prime}$ ' Est, dans le $9^{\mathrm{e}}$ (neuvième) arrondissement au NordOuest de la commune de Cotonou. Elle s'étend sur une superficie d'environ $1400 \mathrm{~m}^{2}$.

Les trois décharges reçoivent des déchets solides ménagers et assimilés pré-collectés dans la ville de Cotonou. Les déchets sont déversés directement sur le sol sans aucun traitement des ordures ni protection du sol, ce sont des exemples de décharges de type sauvage. Outre les déchets ménagers et assimilés, on y trouve aussi des déchets d'équipements électriques et 
électroniques, des pneumatiques, des déchets biomédicaux (le matériel infectieux, pointu ou tranchant tels que les aiguilles, les seringues, les lames, la verrerie et les lamelles de cultures).

Il faut souligner que les déchets collectés dans la ville de Cotonou sont estimés à 500 tonnes sur les 718 tonnes de déchets solides ménagers produits par jour (Mairie Cotonou, 2008). Les 218 tonnes restantes sont rejetés quotidiennement dans des endroits inappropriés sans aucun contrôle.

\section{Méthode d'échantillonnage}

Des échantillons de sol ont été prélevés à l'aide d'une tarière au niveau des trois décharges retenues. Ces échantillons ont été prélevés dans trois horizons sur chaque site : 0 à $10 \mathrm{~cm}$, à $40 \mathrm{~cm}$ puis à $1 \mathrm{~m}$, sauf sur le troisième site où les prélèvements ont été faits dans les $10 \mathrm{~cm}$ et $40 \mathrm{~cm}$ parce que la nappe phréatique se retrouve déjà à $1 \mathrm{~m}$. Pour chaque horizon de prélèvement, des échantillons composites ont été constitués. Au total, huit (8) échantillons composites de 1 kilogramme $(\mathrm{kg})$ de sol pour chaque échantillon ont été constitués.

\section{Méthode d'analyse}

Les analyses effectuées ont porté essentiellement sur les métaux lourds que sont le cadmium, le plomb et le cuivre. Ces analyses ont été réalisées au laboratoire d'hygiène, d'assainissement, d'écotoxicologie et de santé environnementale (HECOTES) à Abomey-Calavi. L'analyse du cadmium a été effectuée suivant la Dithizone Method. Celle du cuivre a été réalisée par la Porphyrin Method et celle du plomb suivant la méthode LeadTrak ${ }^{\mathrm{TM}}$ Fast Column Extraction.

La mesure du $\mathrm{pH}$ a été effectuée par potentiométrie et celle de la matière organique a été déterminée par la perte au feu.

\section{Caractérisation des déchets solides ménagers}

La caractérisation des a été faite afin de connaître leur composition. La méthodologie utilisée est dérivée de la méthode française MODECOM (méthode de caractérisation des ordures ménagères, cette méthode ne pouvant être exactement appliquée pour insuffisance de moyens). Elle comprend l'échantillonnage, le tamisage et le tri des composants, la pesée de chaque composante.

Le traitement des données collectées a été fait au moyen d'outils informatiques (Excel 2013, SPSS 21, Arc GIS 10.3).

\section{Résultats}

\section{Composition des déchets solides ménagers produits à Cotonou}

Les résultats de la caractérisation effectuée sont présentés dans le tableau I. 
Tableau I : Composition des DSM produits à Cotonou en 2017

\begin{tabular}{clc}
\hline Nombres & \multicolumn{1}{c}{ Catégories } & Composants $(\boldsymbol{\%})$ \\
\hline $\mathbf{1}$ & Déchets putrescibles & 31,6 \\
$\mathbf{2}$ & Papiers et cartons & 6 \\
$\mathbf{3}$ & Composites & 0 \\
$\mathbf{4}$ & Textiles et textiles sanitaires & 4,2 \\
$\mathbf{5}$ & Bois & 1,2 \\
$\mathbf{6}$ & Plastiques & 7 \\
$\mathbf{7}$ & Combustibles non classés & 3,2 \\
$\mathbf{8}$ & Verres & 5,4 \\
$\mathbf{9}$ & Métaux & 1 \\
$\mathbf{1 0}$ & Incombustibles non classés & 5,4 \\
$\mathbf{1 1}$ & Déchets ménagers spéciaux & 1,2 \\
$\mathbf{1 2}$ & Eléments fins & 33,8 \\
\hline
\end{tabular}

Source : cette recherche, 2017

Les déchets solides ménagers sont riches en déchets putrescibles (31,6 $\%)$ et en éléments fins $(33,8 \%)$. Cependant, on y retrouve aussi des éléments qui peuvent contenir des métaux lourds (déchets plastiques, verres, déchets ménagers spéciaux tels que les piles, accumulateurs de téléphones portables, médicaments, tubes et ampoules fluorescentes, chargeurs de portable, jouets électriques, vernis à ongles, produits d'hygiène). En considération des résultats de la caractérisation, les déchets solides ménagers ne peuvent être accumulés sur le sol sans aucune mesure de protection préalable puisqu'ils peuvent nuire à l'environnement.

\section{Impacts des décharges sauvages de déchets solides sur les sols}

Les résultats d'analyses des sols sont présentés dans le tableau II

Tableau II Résultats d'analyse des sols des décharges sauvages

\begin{tabular}{|c|c|c|c|c|c|c|c|c|}
\hline \multirow{2}{*}{$\begin{array}{c}\text { Paramètres } \\
\text { (Métaux en } \\
\text { mg/kg) }\end{array}$} & \multicolumn{3}{|c|}{ Site 1} & \multicolumn{3}{|c|}{ Site 2} & \multicolumn{2}{|c|}{ Site 3} \\
\hline & $10 \mathrm{~cm}$ & $40 \mathrm{~cm}$ & $1 \mathrm{~m}$ & $10 \mathrm{~cm}$ & $40 \mathrm{~cm}$ & $1 \mathrm{~m}$ & $10 \mathrm{~cm}$ & $40 \mathrm{~cm}$ \\
\hline pH KCl & 6,490 & 6,600 & 6,610 & 6,810 & 6,490 & 6,580 & 6,610 & 6,420 \\
\hline Matière & 9,50 & 71,70 & 7,30 & 21,30 & 36,80 & 25 & 58,40 & 8,80 \\
\hline Organique $\%$ & & & & & & & & \\
\hline Cadmium & 2,028 & 3,872 & - & 5,777 & 2,640 & $\mathbf{1 , 0 8 0}$ & 6,520 & 1,040 \\
\hline Cuivre & 695,830 & 782,435 & - & 402,390 & 904 & - & 836 & 808 \\
\hline Plomb & 302,190 & 774,500 & 259,481 & 211,155 & 196 & 20 & 192 & 128 \\
\hline
\end{tabular}

Source : la présente recherche, 2019

Les teneurs en éléments traces métalliques varient entre $1,040 \mathrm{mg} / \mathrm{kg}$ et $6,520 \mathrm{mg} / \mathrm{kg}$ pour le cadmium, $402,390 \mathrm{mg} / \mathrm{kg}$ et $904 \mathrm{mg} / \mathrm{kg}$ pour le cuivre, $20 \mathrm{mg} / \mathrm{kg}$ et $774,500 \mathrm{mg} / \mathrm{kg}$ pour le plomb. Le $\mathrm{pH}$ mesuré dans les échantillons varie entre 6,420 et 6,810 , ce ph indique que ces métaux évoluent dans un 
milieu légèrement acide. Les valeurs de la matière organique enregistrées oscillent entre $7,30 \%$ et $71,70 \%$. Ce qui indique que les sols sont riches en matière organique.

Par ailleurs, l'évolution quantitative des paramètres chimiques et des éléments traces métalliques varie en fonction des profondeurs. Ainsi, dans l'horizon superficiel $(10 \mathrm{~cm}$ à $40 \mathrm{~cm})$, il est enregistré une forte concentration de matière organique dans les sols des trois sites. Dans ce même horizon, se concentrent les métaux lourds analysés (cadmium, cuivre, plomb).

A $1 \mathrm{~m}$ de profondeur, il est observé une faible concentration de la matière organique sur le site 1 . Les teneurs des sols en cadmium et en cuivre n'ont pu être déterminées à ce niveau car elles sont très faibles, mais le plomb y présente une concentration élevée $(259,481 \mathrm{mg} / \mathrm{kg})$. A cette profondeur sur le site 2 , il est enregistré une importante concentration de la matière organique, le cadmium et le plomb y sont présents en de faibles quantités, mais le cuivre n'y est pas détecté. Au niveau du troisième site, aucune observation n'a été faite à cette profondeur.

Les concentrations de ces métaux lourds sont comparées aux normes recommandées par le CCME 1999 (Conseil Canadien des Ministres de l'Environnement [CCME], 2007) et AFNOR NF U44-041 (AFNOR NF U44041 cité par Bodjona et al., 2012), (tableau III).

Tableau III : Comparaison des concentrations des métaux lourds des décharges étudiées avec les normes CCME 1999 et AFNOR NF U44-041

\begin{tabular}{|c|c|c|c|c|c|c|c|c|c|c|}
\hline \multirow{2}{*}{$\begin{array}{l}\text { Métaux } \\
\text { (mg/kg) }\end{array}$} & \multicolumn{3}{|c|}{ Site 1} & \multicolumn{3}{|c|}{ Site 2} & \multicolumn{2}{|c|}{ Site 3} & \multirow{2}{*}{$\begin{array}{l}\text { Normes } \\
\text { CCME } \\
\text { (mg/kg) }\end{array}$} & \multirow{2}{*}{$\begin{array}{l}\text { AFNOR } \\
\text { NF U44- } \\
\text { 041 } \\
(\mathrm{mg} / \mathrm{kg})\end{array}$} \\
\hline & $10 \mathrm{~cm}$ & $40 \mathrm{~cm}$ & $1 \mathrm{~m}$ & $10 \mathrm{~cm}$ & $40 \mathrm{~cm}$ & $1 \mathrm{~m}$ & $10 \mathrm{~cm}$ & $40 \mathrm{~cm}$ & & \\
\hline Cadmium & 2,028 & 3,872 & - & 5,777 & 2,640 & 1,080 & 6,520 & 1,040 & 1,4 & 2 \\
\hline Cuivre & 695,830 & 782,435 & - & 402,390 & 904 & - & 836 & 808 & 63 & 100 \\
\hline Plomb & 302,190 & 774,50 & 259,481 & 211,155 & 196 & 20 & 192 & 128 & 70 & 100 \\
\hline
\end{tabular}

Les concentrations des métaux lourds détectés dans les horizons supérieures $(10 \mathrm{~cm}$ et $40 \mathrm{~cm})$ des sols des décharges étudiées dépassent largement les normes CCME 1999 et AFNOR NF U44-041 à l'exception du sol de la troisième décharge qui présente une teneur en cadmium inférieure aux normes.

A $1 \mathrm{~m}$ de profondeur les concentrations des métaux sont inférieures aux normes sur les trois sites sauf celle du plomb qui reste supérieure aux normes $(259,481 \mathrm{mg} / \mathrm{kg})$ sur le site 1 .

\section{Discussion}

Les sols des décharges sauvages étudiées présentent des teneurs élevées en métaux lourds. Cela est probablement dû à l'hétérogénéité des déchets. Les déchets d'équipements électriques et électroniques, les pneumatiques, les matières plastiques et des déchets médicaux présents sur les 
décharges sauvages de déchets ménagers peuvent être sources de la forte concentration des métaux lourds dans les sols (Bouchakor ; Malayeri cités par Bodjona et al., 2012).

Par ailleurs, l'accumulation des métaux lourds enregistrée dans les horizons superficiels des sols $(10 \mathrm{~cm}$ et $40 \mathrm{~cm})$ pourrait être expliquée par la teneur des sols en matière organique et le $\mathrm{pH}$. En effet, la matière organique et le $\mathrm{pH}$ sont des facteurs qui influent sur la mobilité des métaux dans le sol (Hlavackova, 2005 ; Marseille \& Denot, 2007). Ayant une capacité d'échange cationique élevée et d'importante surface spécifique, la matière organique du sol contribue à adsorber de fortes quantités de métaux lourds (Kabata-Pendias, cité par Nduwayezu, 2010). Plusieurs auteurs ont montré que le cadmium, le cuivre et le plomb ont tendance à s'accumuler dans les horizons supérieurs du sol, préférentiellement sur les matières organiques (Adriano cité par Institut National de l'Environnement Industriel et des RISques [INERIS], 2011 ; Benjamin \& Leckie ; Bruemmer \& coll. ; Fu \& coll. cités par Conseil Canadien des Ministres de l'Environnement [CCME], 1999 ; Cecchi, 2008 ; INERIS, 2014 ; INERIS cité par INERIS 2014 ; Laperche, Dictor, ClozelLeloup, \& Baranger, 2004). En outre, l'adsorption des métaux dépend du pH (Sbaa et al. cité par Benahmed, Dellal et Hellal, 2016 ; Hlavackova, 2005). En fait, une augmentation du $\mathrm{pH}$ favorise l'adsorption des métaux. Dans un milieu acide, l'adsorption du plomb et du cuivre peut être observée à $\mathrm{pH} \geq 5$, celle du cadmium à $\mathrm{pH} \geq 6$ (Alloway cité par Cecchi, 2008 ; Citeau cité par Kouamé et al., 2006 ; Pichard et al. cités par Kouamé et al., 2006 ; Marseille \& Denot, 2007). Les pH des sols étudiés, variant entre 6,600 et 6,810 sembles favorables à l'adsorption des métaux.

La concentration élevée du plomb observée en profondeur (1m) sur le site 1 pourrait être due au niveau élevé de la nappe de Cotonou qui varie entre $1 \mathrm{~m}$ et 1,5 m. En effet, une nappe peu profonde et des eaux de surface situées à proximité de la source de contamination augmentent significativement les risques de mobilité et de transport du plomb (Laperche, Dictor, Clozel-Leloup, \& Baranger, 2004). D'autre part, cela peut s'expliquer aussi par les caractéristiques géologiques du site. Le sol du site de Cotonou étant essentiellement sablonneux (80 \% de sables grossiers) avec une porosité supérieure à $40 \%$ et un coefficient d'emmagasinement de 20\% (Gandaho ; Ahoussinou cités par Odoulami, 2009 p. 58). Or, les sols sablonneux possèdent une faible capacité de rétention des métaux lourds cationiques parce qu'ils contiennent en général peu de phyllosilicates pouvant adsorber de fortes quantités de métaux lourds (Kabata-Pendias cité par Nduwayezu, 2010).

D'autre part, les résultats de la caractérisation montrent que les déchets solides ménagers sont riches en matières organiques, et contiennent aussi des déchets composés de substances chimiques et des métaux lourds. Ces résultats s'accordent avec ceux obtenus par Gbèdo (2010). La caractérisation des 
déchets solides ménagers effectuée par celui-ci présente une proportion importante pour déchets putrescibles $(48,51 \%)$, les éléments fins $(25,87 \%)$ et les matières plastiques $(7,74 \%)$. Il faut signaler cependant, une augmentation de la proportion des déchets ménagers spéciaux $(1,2 \%)$ comparativement à $0,29 \%$ obtenu par Gbèdo (2010). Cette évolution dans la composition des déchets peut être attribuée au changement et à l'évolution des modes de consommation.

\section{Conclusion}

Les résultats obtenus dans la présente recherche permettent de conclure que les sols des décharges sauvages sont contaminés par le cadmium, le cuivre et le plomb issus des déchets. La distribution verticale des métaux dans les sols révèle leur accumulation dans les horizons superficiels. Mais le plomb a atteint la profondeur sur certains sites. Il s'avère donc nécessaire de faire des recherches sur la biodisponibilité des métaux pour évaluer leur toxicité. Par ailleurs, comme la plupart des décharges sauvages à Cotonou, les trois décharges étudiées n'ont jamais été l'objet d'étude publiée relative à l'évaluation de la qualité physico-chimique du sol. Au regard des résultats obtenus, il est important de faire des recherches sur d'autres décharges sauvages à Cotonou afin d'évaluer l'état de pollution des sols.

\section{Reference:}

1. Babio S., Baloubi M. D. \& Houssou S. C. (2016). Perceptions des nuisances environnementales liées aux déchets solides ménagers dans les centres urbains du Nord-Bénin: cas des villes de Parakou, Djougou, Kandi et Malanville. European scientific journal, vol 12, n'23, pp 349-365

2. Benahmed M., Dellal A. \& Hellal B. (2016). Mobilite du plomb et du zinc issus de retombées atmosphériques dans le sol : cas de la zone industrielle de Tiaret, Algerie. European scientific journal, vol 12, No.18, pp 131-140

3. Blalogoe C. P. (2009). Problématique de la valorisation agricole des déchets solides ménagers de la ville de Cotonou (Mémoire de Master). Université d'Abomey-Calavi.

4. Bodjona B. M., Kili A. K., Tchegueni S., Kennou B., Tchangbedji G. \& El Meray M. (2012). Evaluation de la quantité des métaux lourds dans la décharge d'Agoè (Lomé-Togo) : cas du plomb, cadmium, cuivre, nickel et zinc. International Journal of Biological and Chemical Science, 6(3): 1368-1380

5. CCME (2007). Indice de la qualité des sols du CCME 1.0. Rapport technique, dans Recommandations canadienne pour la qualité de l'environnement, 1999, Conseil Canadien des Ministres de 
l'Environnement, Winnipeg. Retrieved from www.ccme.ca , 19 /05/2019

6. CCME (1999). Recommandations canadiennes pour la qualité des sols : Environnement et santé humaine — plomb (1999), dans Recommandations canadiennes pour la qualité de l'environnement, 1999, Winnipeg, le Conseil.

7. Gbedo V. (2010). Problématique de la valorisation des déchets plastiques à Cotonou: approche pour une maîtrise des aspects techniques et socio-économique (Thèse de doctorat). Université d'Abomey-Calavi.

8. Hediblè C. S. (2015). Perceptions populaires et gestion des déchets solides ménagers à Agla dans le $13^{\text {ème }}$ arrondissement à Cotonou au Bénin (Afrique de l'Ouest). Revue de géographie du laboratoire LeïdiISSN 0851-2515, ${ }^{\circ} 13$, décembre, pp 205-217. Retrieved from www.ugb.sn , 27/08/2017

9. Hlavackova P. (2005). Evaluation du comportement du cuivre et du zinc dans une matrice de type sol à l'aide de différentes méthodologies (Doctoral dissertation). Institut National des Sciences Appliquées de Lyon, Lyon.

10. INERIS (2014). Données technico-économiques sur les substances chimiques en France : cuivre, composés et alliages, DRC-14-13688102236A. Retrieved from http://rsde.ineris.fr/, 29/04/2019

11. INERIS (2011). Fiche de données toxicologiques et environnementales des substances chimiques : cadmium et ses dérivés.

12. INSAE (2016). Cahier des villages et quartiers de ville du département du littoral, 20 p. Retrieved from https://www.insae-bj.org , 20/08/2017

13. Kouame K. I., Gone L. D., Savane I., Kouassi A. E., K. Koffi K., Goula A. T. B. \& Diallo M. (2006). Mobilité relative des métaux lourds issus de la décharge d'Akouédo et risque de contamination de la nappe du Continental Terminal (Abidjan - Côte d'Ivoire). Afrique Science, 02(1), 39-56.

14. Laperche V., Dictor C. M., Clozel-Leloup B., \& Baranger P. (2004). Guide méthodologique du plomb appliqué à la gestion des sites et des sols pollués. BRGM/RP 52881-FR, Rapport final. Retrieved from www.infoterre.brgm.fr , 13/07/2019

15. Marseille F. \& Denot A. (2007). Mobilité et biodisponibilité des contaminants présents dans les sols aux abords des infrastructures et impact sur la santé. Rapport intermédiaire. Retrieved from www.lara.inist.fr , 13/07/2019

16. Mairie de Cotonou (2008). Plan de développement de la ville de Cotonou. DPDM. Retrieved from www.ancb-benin.org , 20/05/2016 
17. Matech F., Zaakour F., Moustarhfer K., Chemsi Z., Benazzouz I. \& Saber N. (2014). Concentrations en éléments traces métalliques dans les sols irrigués par les eaux usées versées dans l'oued merzeg (casablanca -maroc). European Scientific Journal, vol.10, n²9, pp. 1857-7881

18. Nduwayezu I. (2010). Adsorption et désorption du plomb dans un sol sablonneux traité par divers amendements (Mémoire de Maîtrise). Université du Québec à Montréal.

19. Nhari F., Sbaa M., Vasel J. L., Fekhaoui M. \& El Morhit M. (2014). Contamination des sols d'une décharge non contrôlée par les métaux lourds : cas de la décharge Ahfir-Saidia (Maroc Oriental). J. Mater. Environ. Sci. 5(5) : 1477-1484.

20. Odoulami L. (2009). La problématique de l'eau potable et la santé humaine dans la ville de Cotonou (Thèse de Doctorat). Université d'Abomey-Calavi.

21. PNUD (2011). Diagnostic de la gestion des déchets au Bénin. Unpublished document.

22. Thonart P., Diabate S. I., Hiligsmann S. \& Lardinois M. (2005). Gestion des déchets ménagers et sites d'enfouissement technique dans les pays du Sud. Guide pratique. Retrieved from www.iepf.org, $04 / 01 / 2018$ 